\section{Before Mutation}

Selected

A

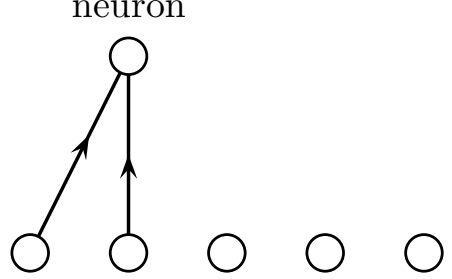

Rest of the neurons within neuronal group

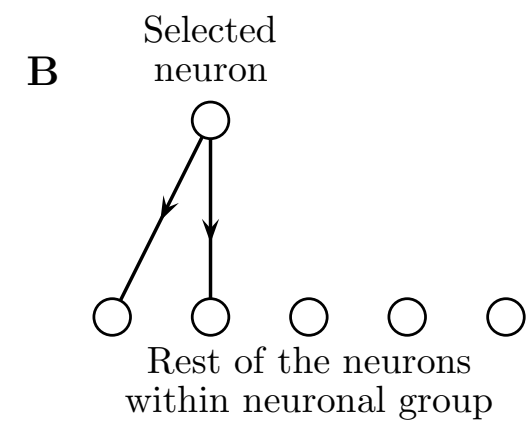

C

Selected

neuron

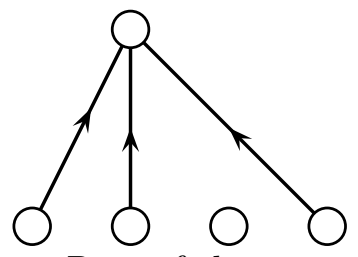

Rest of the neurons within neuronal group

D

Selected

neuron

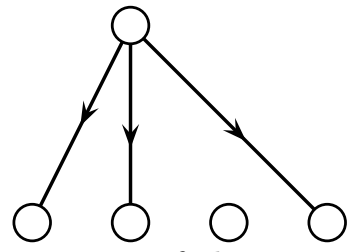

Rest of the neurons within neuronal group
After Mutation

Selected

neuron

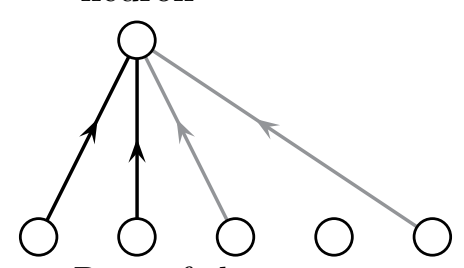

Rest of the neurons

within neuronal group

Selected

neuron

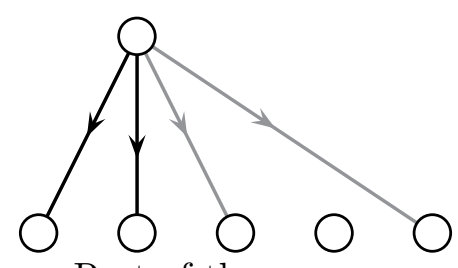

Rest of the neurons

within neuronal group

Selected

neuron

$O$

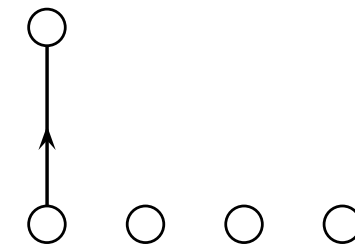

Rest of the neurons within neuronal group

Selected

neuron

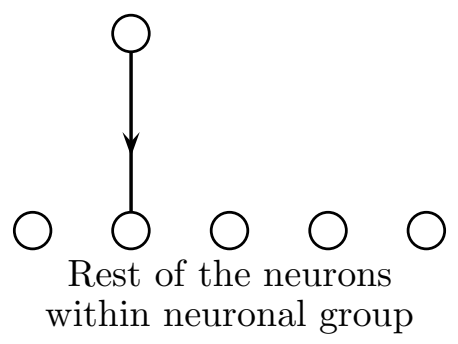

